\title{
A Novel Comparative Evaluation for the Quality of Oral Generic Drugs
}

\author{
Masato Terashita, Kazuhiro Iguchi, Shigeyuki Usui and Kazuyuki Hirano* \\ Laboratory of Pharmaceutics, Department of Medical Pharmaceutics, Gifu Pharmaceutical University \\ $\left[\begin{array}{l}\text { Received March 27, } 2012 \\ \text { Accepted December 3, } 2012\end{array}\right]$
}

\begin{abstract}
Anxieties about the quality of generic drugs are one of reasons why these drugs are not generally used in therapies in Japan. To reduce anxieties, we propose a statistical and objective method to evaluate the quality of generic drugs using the coefficient of variances (CVs) calculated from their pharmacokinetic parameters. CVs were estimated from AUC, Cmax, Tmax, t1/2, and MRT, which were provided as publicly-available drug information. The generic drug assessment tool (G-DAT), an original equation derived from the ratio of CVs of corresponding generic to original drugs, and the quality of generic drugs was statistically evaluated. As expected, when evaluated with G-DAT, there was little variance between original and generic drugs using 11 medicines. Antihypertensive dihydropyridines with a lower solubility were shown to have a larger CV. No significant difference in CVs was observed when famotidine and ebastine OD tablets were taken with or without water. G-DAT and the ratio of $\mathrm{CVs}(\mathrm{CCVs})$ of generic to original drugs revealed pharmacokinetic equivalences between generic and original drugs. The proposed comparative evaluation showing equivalent pharmacokinetics of generic to original drugs can be used as one solution to reduce anxieties and increase the reliability of generic drugs.
\end{abstract}

Key words — bioequivalence, biopharmaceutics classification system (BCS), generic tablet, pharmacokinetics, vector space model

\section{Introduction}

The cost of medical care in Japan keeps increasing year after year due to rapid aging of the population resulting from a decline in the birthrate. A major policy change for maintaining the health-insurance system that covers all of its citizens has been taken in order to cut down the cost. ${ }^{1}$ The Ministry of Health, Labour, and Welfare in Japan has implemented multilateral policies for medical treatment fees. To reduce costs, a preferential policy for the increased use of generic drugs, which have a lower usage in Japan than Europe and the United States, is being carried out. An institutional revision began in 2002 to take on additional medical fees for prescribing and dispensing generic drugs. In a revision in
2006, physicians started to advise patients of generic drugs that could be used as replacements for original drugs in the remarks column of the prescriptions.

As the quality of generic drugs keeps improving, the prescription of generic drugs has been promoted. Drug safety and bioequivalence studies for healthy adults were problems that needed to be addressed on the application for approval of the production of generic drugs before 1997. Guidelines on the bioequivalence study of generic drugs were formulated by the former Ministry of Health, and Welfare in 1997. In these guidelines, a dissolution test was added to the revised bioequivalence study. All generic drugs, which were put on the market before and after 1997, underwent a re-evaluation of drug quality according to

* 1-25-4, Daigaku-nishi, Gifu, 501-1196 Japan 
these revised guidelines. The results of the study were reported in the orange book, a data book of drug information used therapeutically in Japan. ${ }^{2}$

Previously, generic drugs had not been used in therapy, however policy changes for the use of these drugs were promoted. The reasons why generic drugs were not generally used were anxieties about the quality of the drugs, lack of drug information, and an unsteady supply of the drugs. These reasons have not been overcome completely and physicians may not fully understand the processes on approval of generic drugs and test contents in the orange book. The lack of reliability of the therapeutic effects of generic drugs, depending on the quality, caused physicians to hesitate in using these drugs. ${ }^{3)}$ Since the patents of some oral medicines in therapeutic use expired after 2010, the generic drugs of these off-patent medicines will be produced and put on the market in the near future. This may possibly result in increased opportunities for the use of these generic drugs. Therefore, it is necessary to reduce anxieties about the quality of generic drugs in order to increase opportunities for their prescription.

We strive torwards a statistical and objective method to evaluate the quality of generic drugs in order to reduce anxieties and increase the use of these drugs. In this study, we propose an analytical method that provides relative value calculated from the coefficient of variance for original drugs and corresponding generic drugs. The coefficient of variance was estimated from pharmacokinetic parameters: area under the curve (AUC), maximum concentration (Cmax), time required for Cmax (Tmax), half-life (t1/2), and mean residence time (MRT), which were provided in the orange book as publicly-available drug information, and the variances obtained from corresponding generic drugs were assessed. Furthermore, we discuss the correlation between the solubility and coefficient of variance of several drugs.

\section{Methods}

\section{Pharmacokinetic parameters}

Pharmacokinetic parameters, AUC, Cmax, Tmax, and $\mathrm{t} 1 / 2$, of a general drug were referred in the orange book as publicly-available drug information. AUC and Cmax are essential parameters for approval of generic drugs, but Tmax and $\mathrm{t} 1 / 2$ are parameters which are recommended to present in publicly-available drug information. These parameters are provided as a database from Gifu Pharmaceutical University. A pharmacokinetic parameter MRT, if available, was taken instead of t1/2 for a sustained-release medicine.

\section{Evaluations for the variance of the quali- ty between original and generic drug}

A vector space model derived from the vector space method is used to retrieve the similarity of information on World Wide Web. ${ }^{4}$ The pharmacokinetic characteristics of original and generic drugs were assumed as information of the drugs and represented as a vector calculated with the pharmacokinetic parameters of the drugs. The model was applied to compare the similarity between original and generic drugs. The Euclidean vector is a geometric entity endowed with magnitude (or length) and direction. The similarity of two vectors, vectors $A$ and $B$, in the vector space method generally corresponds to an angle formed by vectors A and B and is calculated with the cosine theorem. However, the vector from the original drug was based on " 1 " for simplicity in the proposed method since the original drug is compared to the generic drug. Therefore, the similarity between original and generic drugs was esti- 
mated as length and direction of the two vectors from the original and generic drugs. Equations estimated from pharmacokinetic parameters of a drug as a vector are indicated below.

The mean and the standard deviation were estimated from the pharmacokinetic parameters of the drugs, AUC, Cmax, Tmax, and t1/2 or MRT, and the coefficient of variance $(\mathrm{CV})$ was calculated by dividing the standard deviation by the mean. The $\mathrm{CV}$ ratio $(\mathrm{CCV})$ of generic to original drugs was calculated using equation 1 which estimates the variance of pharmacokinetic parameters between standard and generic drugs. Even if the standard error was used to calculate CCV instead of the standard deviation, the CCV was estimated as equal to that with the standard deviation as shown in equation 1.

$C C V=\frac{C V(\text { parameter from generic drugs (paramerer; } g))}{C V(\text { parameter from standard drugs (paramerer; } s))}$ Example: $C C V(A U C)=\frac{C V(A U C ; g)}{C V(A U C ; s)}$

The pharmacokinetic parameters: AUC, Cmax, Tmax, $\mathrm{t} 1 / 2$ and MRT, indicate the pharmacokinetic characteristics of the drug. The CCV of these parameters represents the range of variance of the parameter between generic and original drugs. Therefore, two- and four-dimensional vector equations settled CCVs as variables that represent variations of pharmacokinetic characteristics from generic drugs. The generic drug assessment tool (G-DAT) shown as equation 2 represents the vector equation to estimate the variations as length. G-DAT(2) is estimated with essential pharmacokinetic parameters, AUC and Cmax, and, if available, G-DAT(4) with AUC, Cmax, Tmax, and $\mathrm{t} 1 / 2$ (or MRT) is calculated because of its accuracy improvement. Denominators use $\operatorname{root}(2)$ and $\operatorname{root}(4)=2$ for G-DAT(2) and
G-DAT(4), respectively, to obtain the range of variance based on 1, where G-DAT(2) and GDAT(4) are calculated using two essential parameters, AUC and Cmax, and all four parameters, AUC, Cmax, Tmax, and t1/2 or MRT, respectively.

$G-D A T(2)=\frac{\sqrt{(C C V(A U C))^{2}+(C C V(C m a x))^{2}}}{\sqrt{2}}$
$G-D A T(4)=\frac{\sqrt{(C C V(A U C))^{2}+(C C V(C m a x))^{2}+(C C V(T m a x))^{2}+(C C V(t 1 / 2))^{2}}}{2}$

Since the bioequivalence study of sustained-release generic drugs was performed under both fasting and feeding conditions, G-DAT of these drugs was estimated using equation 3 which includes the data obtained from both of these conditions. In equation $3, \mathrm{bm}$ and am represent fasting and feeding, respectively.

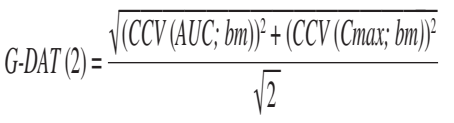

$$
\begin{aligned}
& +\frac{\sqrt{\left((C C V(\operatorname{AUC} ; \text { am }))^{2}+(\operatorname{CCV}(\operatorname{Cmax} ; \text { am }))^{2}\right.}}{\sqrt{2}} \\
& G \cdot D A T(4)=\frac{\sqrt{\left.(C C V(A U C ; ~ b m))^{2}+(C C V(C m a x ; ~ b m))^{2}+(C C V(\operatorname{Tmax} ; \text {; } m))^{2}+(C C V(11) ; \text {; } m)\right)^{2}}}{2}
\end{aligned}
$$

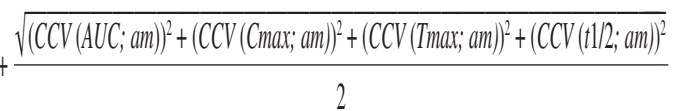

G-DAT estimating the distance between the vectors from original and generic drugs represents the length of a vector from a generic drug since the length from an original drug is based on 1 . In order to consider the direction of a vector, pharmacokinetic parameters and their corresponding CCV were plotted on horizontal and vertical axes, respectively, to graphically show the direction of a vector containing multiple-dimensions that correspond to pharmacokinetic parameters on a plane. 


\section{Analysis of the correlation between solu- bility and CV}

Pharmacokinetic parameters obtained by the administration of more than two tablets in the bioequivalence study were excluded from analysis because variances may become larger. The parameters represented as the standard error were also avoided. The parameters from an unmetabolite were adopted even if active metabolites were observed since pharmaceutical vendors rarely provide the pharmacokinetic parameters of metabolites.

\section{Results}

\section{Comparison of generic drugs by G-DAT}

G-DATs of amlodipine besylate $5 \mathrm{mg}$ tablets marketed by thirty-three pharmaceutical vendors are listed in Table 1. Thirty-three G-DATs did not show different values and ten of the equal values were observed. The pharmaceutical vendors were given permission by a revision in the Pharmaceutical Affairs Act in 2005 to label generic drugs as their own brands even though the vendors did not make the medicine and sold the product purchased from a pure-play company focused on manufacturing. A drug produced by a manufacturer was put on the market by several pharmaceutical vendors who gave it their own brand names. Therefore, thirty-three tablets counted by brand name were gathered from sixteen manufacturers, and the kinetic parameters of the bioequivalence were compared.

Ninety \% confidence interval of the mean of AUC and Cmax of generic to original drugs is estimated within the range of 0.8 to 1.25 in Japan and the United States. Even if ratios were observed within this range, pharmacists and physicians still recognized product variations of gener-
Table 1 Generic drug assessment tool (G-DAT) of amlodipine $5 \mathrm{mg}$ tablets

\begin{tabular}{|c|c|c|c|c|}
\hline Vendor & Manufacturer & $\begin{array}{c}\text { Number of } \\
\text { samples }\end{array}$ & G-DAT(4) & G-DAT(2) \\
\hline 01 & $\mathrm{a}$ & 12 & 0.9863 & 0.6082 \\
\hline 02 & $\mathrm{a}$ & 12 & 0.9863 & 0.6082 \\
\hline 03 & $\mathrm{~b}$ & 10 & 1.2015 & 0.6492 \\
\hline 04 & $\mathrm{~b}$ & 10 & 1.2014 & 0.6492 \\
\hline 05 & $\mathrm{~b}$ & 10 & 1.2014 & 0.6492 \\
\hline 06 & $\mathrm{~b}$ & 10 & 1.2015 & 0.6492 \\
\hline 07 & $\mathrm{c}$ & 15 & $*$ & 0.6840 \\
\hline 08 & d & 12 & 0.9577 & 0.7350 \\
\hline 09 & $\mathrm{e}$ & 24 & 0.9104 & 0.8384 \\
\hline 10 & $\mathrm{f}$ & 15 & 0.9091 & 0.8709 \\
\hline 11 & $\mathrm{f}$ & 15 & 0.9160 & 0.8709 \\
\hline 12 & $f$ & 15 & * & 0.8710 \\
\hline 13 & $\mathrm{~g}$ & 20 & 1.0952 & 0.9196 \\
\hline 14 & $\mathrm{~g}$ & 20 & 1.0952 & 0.9196 \\
\hline 15 & $\mathrm{~g}$ & 20 & 1.0952 & 0.9196 \\
\hline 16 & $\mathrm{~h}$ & 10 & 0.8157 & 0.9620 \\
\hline 17 & $\mathrm{i}$ & 9 & 1.0110 & 1.0285 \\
\hline 18 & $\mathrm{i}$ & 9 & 1.0110 & 1.0285 \\
\hline 19 & $\mathrm{j}$ & 16 & 0.9021 & 1.0577 \\
\hline 20 & $\mathrm{k}$ & 20 & 0.9496 & 1.0956 \\
\hline 21 & $\mathrm{k}$ & 20 & 0.9496 & 1.0956 \\
\hline 22 & 1 & 12 & 1.1363 & 1.1495 \\
\hline 23 & 1 & 12 & 1.1363 & 1.1495 \\
\hline 24 & 1 & 12 & 1.1502 & 1.1768 \\
\hline 25 & $\mathrm{~m}$ & 16 & 1.0716 & 1.1842 \\
\hline 26 & $\mathrm{n}$ & 20 & 1.0262 & 1.2287 \\
\hline 27 & $\mathrm{n}$ & 20 & 1.0262 & 1.2287 \\
\hline 28 & $\mathrm{n}$ & 20 & 1.0262 & 1.2287 \\
\hline 29 & o & 9 & 1.4299 & 1.2917 \\
\hline 30 & o & 9 & 1.4299 & 1.2917 \\
\hline 31 & o & 9 & 1.4299 & 1.2917 \\
\hline 32 & $\mathrm{p}$ & 12 & 1.2069 & 1.5124 \\
\hline 33 & $\mathrm{p}$ & 12 & 1.2107 & 1.5185 \\
\hline
\end{tabular}

* Not determined because of unavailable parameters.

ic drugs showing differences in the therapeutic effect. Therefore, G-DAT was designed as an assessment tool for the variations of generic drugs. The frequency distribution for G-DATs of amlodipine besylate and loxoprofen sodium hydrate is shown in Fig. 1. Since G-DAT represents an indicator for the variance of generic to original drugs, the larger the G-DAT of a generic drug, the less bioequivalence there is to the original drug. One product each was observed to have more than 1.3 G-DAT in both amlodipine and loxoprofen tablets. G-DATs for generic drugs approved according to the guidelines after 1997 are listed 


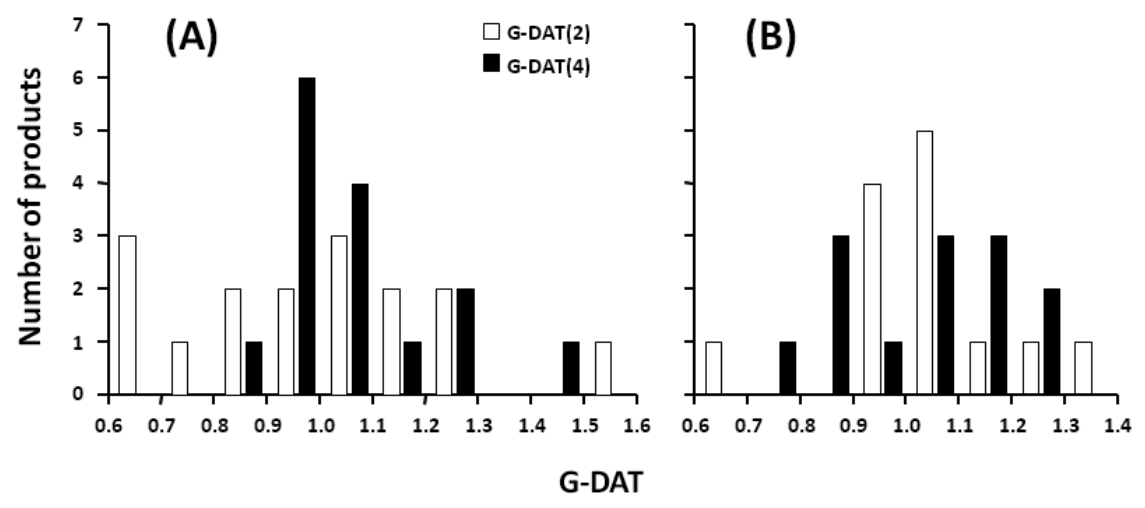

Fig. 1 Frequency distribution of G-DAT(2) and G-DAT(4) from (A) amlodipine and (B) loxoprofen tablets

G-DAT of 16 amlodipine and 13 loxoprofen tablets was calculated with equation 2.

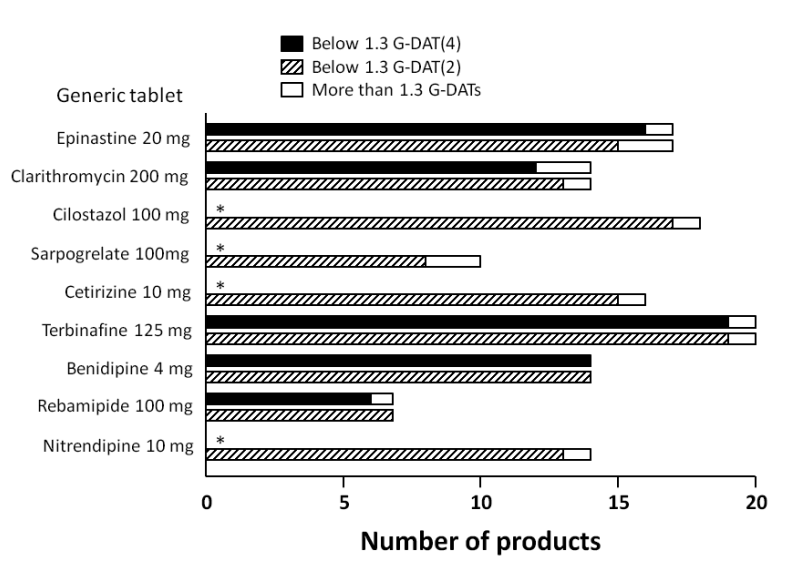

Fig. 2 Number of generic products showing below and more than 1.3 G-DATs

G-DAT of generic tablets was calculated with equation 2. Number of products showing below and more than $1.3 \mathrm{G}-\mathrm{DAT}$ were expressed.

* Not determined.

in Fig. 2. Most of the products were below 1.3 G-DAT although one or two of the products were observed to have more than 1.3 G-DAT. If the mean of $\mathrm{CCV}$ from the kinetic parameters was more than 1.3 in equation 2, G-DAT is calculated to be more than 1.3 . Ninety $\%$ confidence interval of the mean is required to be within a range of 0.8 to 1.25 as described above. A CCV value of 1.3 is estimated if the ratio of the mean is over 1.3 , although caution is advised when simply comparing $\mathrm{CCV}$ with the mean because $\mathrm{CCV}$ includes a concept of variance. Therefore, $1.3 \mathrm{G}$ DAT appeared to be a criterion value for judging the quality of generic drugs. These results sug- gest that the variances for generic to original drugs are generally less than that expected when evaluated with 1.3 G-DAT.

Significant values of the kinetic parameter written in the drug package insert were not standardized in some cases in Japan when the parameters were referenced in the insert. Bioequivalence data for a generic drug made by a single manufacturer are supposed to be the same even if some vendors market it with their own brand names. Even though A and B venders, for example, were assumed to carry a generic drug from a single manufacturer, Tmax values, 1.95 and 2.04, for the generic and the original drug, respectively, were represented, in A's insert and Tmax values, 2.0 and 2.0, were represented in B's insert. G-DATs with these parameters represent two different values and two drugs, A and B, are judged to be different products. Furthermore, the kinetic parameters, Tmax and $t 1 / 2$, are sometimes not shown in the insert although AUC and Cmax are necessary, and the parameters from the original drugs were also sometimes absent from the insert. Thus, some inadequacies exist in the format of the drug package insert. Since the bioequivalence study for generic drugs is required to confirm the equivalence of the kinetic parameters between original and generic drugs, it is desirable to have all data 
of the study presented in the insert. A contentrich insert could increase reliability of generic drugs because pharmacists and other medical staff frequently refer to it.

\section{CVs affected by water solubility of ge- neric drugs}

The CVs of AUC and Cmax for loxoprofen sodium hydrate and manidipine hydrochloride, which are water soluble and practically insoluble, respectively, are shown in Table 2. The CVs of AUC and Cmax for manidipine were significantly higher than those for loxoprofen.

To further investigate the correlation between solubility and $\mathrm{CV}$, antihypertensive dihydropyridines, as multiproducts, were analyzed (Table $\mathbf{3}$ ). Although these compounds have a common skeleton, their solubility is not the same, being slightly soluble and practically insoluble, because of different side chains. CVs of AUC and Cmax for amlodipine besylate were the smallest in the drugs tested. CVs of AUC and Cmax for benidipine hydrochloride, nilvadipine, nitrendipine, and manidipine hydrochloride, and nicardipine hydro- chloride, respectively, were significantly higher than those of amlodipine. These results suggest that drugs with lower the solubilities have larger CV.

The biopharmaceutics classification system (BCS) is a guide for predicting the intestinal drug absorption proposed by $\mathrm{Yu}$ et al. ${ }^{5)}$ and restricts the prediction using the parameters solubility and intestinal permeability. All those factors are highly important, since $85 \%$ of the most sold drugs in the USA and Europe are orally administered. According to the BCS, drug substances are classified as class I (high permeability, high solubility), class II (high permeability, low solubility), class III (low permeability, high solubility), and IV (low permeability, low solubility). Dihydropiryridines listed in Table $\mathbf{3}$ were classified as class I and II based on the solubility and the permeability of drugs in the BCS. Therapeutics Systems Research Laboratories (TSRL), Inc. provides a BCS service where the class of the drug can be confirmed on the web (URL, http:// www.tsrlinc. com/resources/services/). CVs of AUC and Cmax for the drugs in class II were apparently higher

Table 2 Coefficient of variance (CV) for AUC and Cmax from loxoprofen and manidipine tablets

\begin{tabular}{cccc}
\hline \hline Generic drug & Number of genetics & CV (AUC) & CV (Cmax) \\
\hline Loxoprofen 60 mg tablet & 26 & $0.1815 \pm 0.0500$ & $0.2550 \pm 0.0584$ \\
Manidipine 20 mg tablet & 18 & $0.4789 \pm 0.2155 * *$ & $0.4640 \pm 0.1529 * *$ \\
\hline CV values represent the Mean \pm SD of the samples indicated. & \\
$* * P<0.01$ vs CV from loxoprofen &
\end{tabular}

Table 3 Coefficient of variance (CV) for AUC and Cmax from dihydropirydine tablets

\begin{tabular}{|c|c|c|c|c|c|}
\hline $\begin{array}{l}\text { Generic drug of } \\
\text { dihydropiryridines }\end{array}$ & $\begin{array}{l}\text { Number of } \\
\text { samples }\end{array}$ & Solubility in water & BCS class & $\mathrm{CV}(\mathrm{AUC})$ & $\mathrm{CV}(\mathrm{Cmax})$ \\
\hline Amlodipine $5 \mathrm{mg}$ tablet & 30 & slightly soluble & class I & $0.2404 \pm 0.0605$ & $0.2206 \pm 0.0546$ \\
\hline Nicardipine $20 \mathrm{mg}$ tablet & 16 & slightly soluble & class I & $0.3379 \pm 0.1663$ & $0.3806 \pm 0.1639 * *$ \\
\hline Benidipine $4 \mathrm{mg}$ tablet & 28 & practically insoluble & class II & $0.5323 \pm 0.2949 * *$ & $0.4537 \pm 0.1366 * *$ \\
\hline Nilvadipine $4 \mathrm{mg}$ tablet & 6 & practically insoluble & class II & $0.4221 \pm 0.1031 * *$ & $0.5097 \pm 0.1542 * *$ \\
\hline Nitrendipine $10 \mathrm{mg}$ tablet & 20 & practically insoluble & class II & $0.4997 \pm 0.1910 * *$ & $0.4341 \pm 0.1798 * *$ \\
\hline Manidipine $20 \mathrm{mg}$ tablet & 18 & practically insoluble & class II & $0.4789 \pm 0.2155 * *$ & $0.4630 \pm 0.1529 * *$ \\
\hline
\end{tabular}

$\mathrm{CV}$ values represent the Mean $\pm \mathrm{SD}$ of the samples indicated.

$* * P<0.01$ vs $\mathrm{CV}$ from amlodipine 
than those in class I. Thus, drug solubility affects the CVs of the pharmacokinetic parameters, AUC and Cmax.

To further investigate the solubility of drugs, the dissolution test of the dihydropyridine tablets, amlodipine besylate, benidipine hydrochloride, and manidipine hydrochloride, was performed (Fig. 3). Since these dihydropyridines exhibit weakly-basic characteristics, more than $80 \%$ of the drug component in these tablets dissolved within $30 \mathrm{~min}$ at $\mathrm{pH}$ 1.2. In contrast, manidipine hydrochloride was not dissolved, and about $40 \%$ and $65 \%$ of benidipine hydrochloride and amlodipine besylate, respectively, in these tablets dissolved after 2 hours incubation at $\mathrm{pH} 6.8$.

The solubility of the drug affecting the CVs of kinetic parameters was confirmed with itraconazole, levofloxacin hydrate, and tosufloxacin tosilate tablets. Itraconazole is classified as class II by the BCS, and is weakly-basic and practically insoluble in water. $\mathrm{CV}$ s of $\mathrm{AUC}$ and $\mathrm{Cmax}$ for itraconazole tablets were $0.4056 \pm 0.1734$ and $0.4250 \pm 0.1070$ (mean $\pm \mathrm{SD})$, respectively, with sixteen samples. These values are relatively higher than those for amlodipine besylate, which is classified as class I, and are similar to those for dihydropyridines belonging to class II. Dissolu-

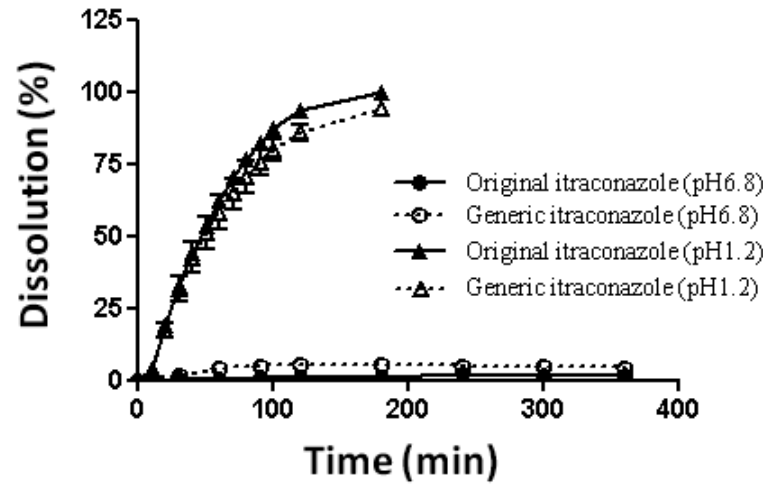

Fig. 4 Dissolution curves of itraconazole tablets Dissolution test was performed according to the method described in the Japanese Pharmacopoeia, Fifteenth Edition.

tion curves for itraconazole tablets at $\mathrm{pH} 1.2$ and 6.8 were shown in Fig. 4. Although good dissolution of itraconazole from the tablet was observed at $\mathrm{pH} 1.2$, most of the component was not dissolved at $\mathrm{pH}$ 6.8. Levofloxacin hydrate shows higher solubility in water than tosufloxacin tosilate. CVs of AUC and Cmax for tosufloxacin were significantly higher than those for levofloxacin, as shown in Table 4.

\section{CVs for orally-disintegrating tablets tak- en with or without water}

The kinetic parameters of orally-disintegrating tablets (ODTs), taken with or without water, were investigated in the bioequivalent study. CVs of AUC and Cmax for ODTs of famotidine and

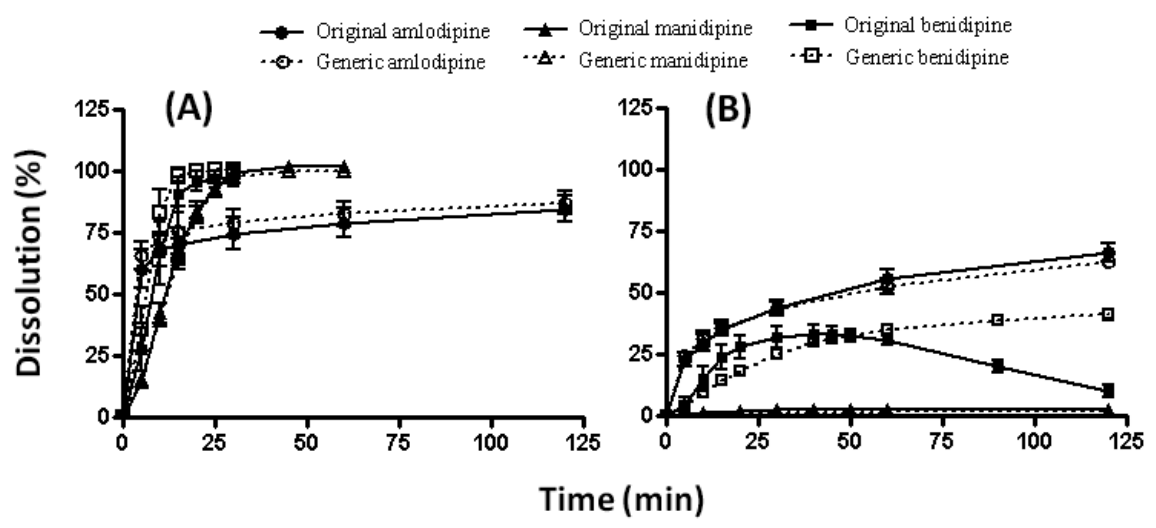

Fig. 3 Dissolution curves of dihydropirydine tablets at (A) $\mathrm{pH} 1.2$ and (B) $\mathrm{pH} 6.8$ Dissolution test was performed according to the method described in the Japanese Pharmacopoeia, Fifteenth Edition. 
Table 4 Coefficient of variance (CV) for AUC and Cmax from levofloxacin and tosufloxacin tablets

\begin{tabular}{cccc}
\hline \hline Generic drug & Number of samples & CV(AUC) & CV(Cmax) \\
\hline Levofloxacin 100 mg tablet & 28 & $0.1400 \pm 0.0329$ & $0.2146 \pm 0.0589$ \\
Tosufloxacin 150mg tablet & 14 & $0.3496 \pm 0.0693 * *$ & $0.3147 \pm 0.0624$ \\
\hline
\end{tabular}

$\mathrm{CV}$ values represent the Mean $\pm \mathrm{SD}$ of the samples indicated.

$* * P<0.01$ vs $\mathrm{CV}$ from levofloxacin

Table 5 Coefficient of variance (CV) for AUC and Cmax from normal and orally-disintegrating tablets of famotidine and ebastine, taken with or without water

\begin{tabular}{|c|c|c|c|c|c|c|}
\hline Generic drug & & Dosage form & $\begin{array}{c}\text { Number of } \\
\text { samples }\end{array}$ & & With water & Without water \\
\hline \multirow{8}{*}{ Famotidine } & \multirow{4}{*}{$10 \mathrm{mg}$} & \multirow{2}{*}{ normal tablet } & \multirow{2}{*}{8} & CV(AUC) & $0.2437 \pm 0.0408$ & \\
\hline & & & & $\mathrm{CV}(\mathrm{Cmax})$ & $0.2856 \pm 0.0332$ & \\
\hline & & \multirow{2}{*}{ OD tablet } & \multirow{2}{*}{10} & CV(AUC) & $0.2620 \pm 0.0482$ & $0.2388 \pm 0.2404$ \\
\hline & & & & $\mathrm{CV}(\mathrm{Cmax})$ & $0.2809 \pm 0.0862$ & $0.2616 \pm 0.2703$ \\
\hline & \multirow{4}{*}{$20 \mathrm{mg}$} & \multirow{2}{*}{ normal tablet } & \multirow{2}{*}{24} & CV(AUC) & $0.2573 \pm 0.0497$ & \\
\hline & & & & $\mathrm{CV}(\mathrm{Cmax})$ & $0.2804 \pm 0.0507$ & \\
\hline & & \multirow{2}{*}{ OD tablet } & \multirow{2}{*}{14} & CV(AUC) & $0.2618 \pm 0.0757$ & $0.2352 \pm 0.0559$ \\
\hline & & & & $\mathrm{CV}(\mathrm{Cmax})$ & $0.2525 \pm 0.0658$ & $0.2676 \pm 0.0834$ \\
\hline \multirow{8}{*}{ Ebastine } & \multirow{4}{*}{$5 \mathrm{mg}$} & \multirow{2}{*}{ normal tablet } & \multirow{2}{*}{10} & CV(AUC) & $0.2468 \pm 0.0478$ & \\
\hline & & & & $\mathrm{CV}(\mathrm{Cmax})$ & $0.2734 \pm 0.0505$ & \\
\hline & & \multirow{2}{*}{ OD tablet } & \multirow{2}{*}{8} & CV(AUC) & $0.3610 \pm 0.1036$ & $0.3427 \pm 0.0174$ \\
\hline & & & & $\mathrm{CV}(\mathrm{Cmax})$ & $0.4052 \pm 0.0776$ & $0.3576 \pm 0.0526$ \\
\hline & \multirow{4}{*}{$10 \mathrm{mg}$} & \multirow{2}{*}{ normal tablet } & \multirow{2}{*}{14} & $\mathrm{CV}(\mathrm{AUC})$ & $0.2924 \pm 0.0577$ & \\
\hline & & & & $\mathrm{CV}(\mathrm{Cmax})$ & $0.3238 \pm 0.0713$ & \\
\hline & & \multirow{2}{*}{ OD tablet } & \multirow[b]{2}{*}{8} & CV(AUC) & $0.3166 \pm 0.0797$ & $0.2618 \pm 0.0689$ \\
\hline & & & & $\mathrm{CV}(\mathrm{Cmax})$ & $0.3076 \pm 0.0791$ & $0.3014 \pm 0.1049$ \\
\hline
\end{tabular}

$\mathrm{CV}$ values represent the Mean $\pm \mathrm{SD}$ of the samples indicated.

ebastine tablets were estimated with the available data (Table 5). If ODTs are taken without water, poor dissolution of the tablets is more likely to occur, and resulting in fluctuating absorption of the drugs. Therefore, it was speculated that CVs without water were larger than those with water. However, significant differences in CVs of ODTs taken with or without water were not observed. This suggests the equivalence of the therapeutic effect of these ODTs, even if taken them with or without water. These ODTs are effective for patients with restricted water intake, or when water is unavailable, although ODTs should be taken with water.

\section{Evaluation of generic drugs}

The quality of generic drugs, rebamipide and furconazole tablets, were compared with G-DAT and $\mathrm{CCV}$ from their pharmacokinetic parameters (Fig. 5). G-DATs of rebamipide tablets from seven vendors ranged from 0.8 to 1.6 , and five out of seven products were from 0.8 to 1.0. Most CCVs indicated about 1.0, similar to those from the original drug, except that one $\mathrm{CCV}(\mathrm{t} 1 / 2)$ was over 2.6. Results from rebamipide tablets suggest that the pharmacokinetic behavior of these generic drugs, except one product, is very similar to that of the original drug and an equivalent therapeutic effect is expected. On the other hand, G-DATs of furconazole tablets from five vendors varied widely from those of rebamipide tablets. CCVs values other than 1 were observed and the shape of the graph presented kinks although they were from two products indicated by G-DAT nearly equal to 1 similar to those from the original drug. In this case, it has been suggested that the thera- 
(A)
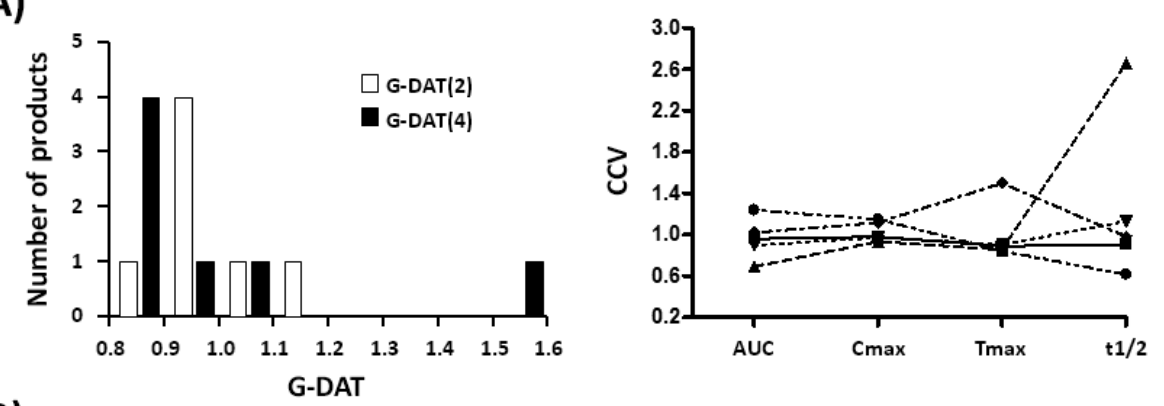

(B)
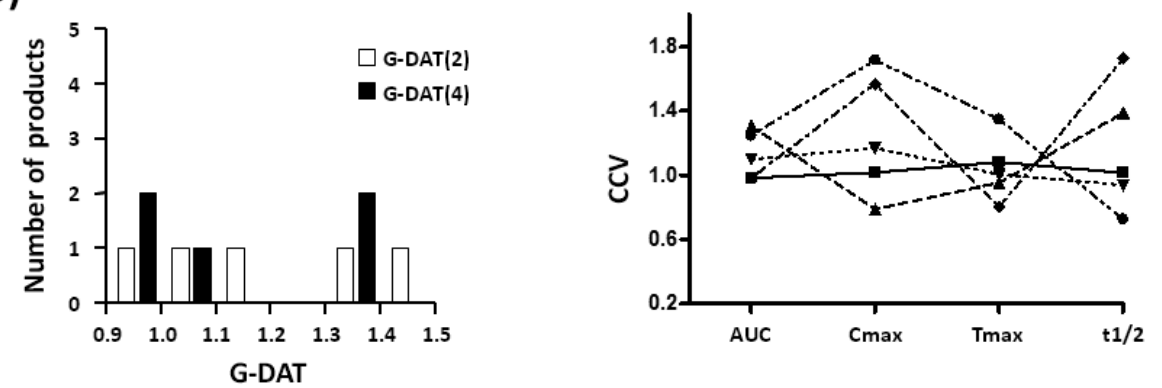

Fig. 5 Evaluation of the quality of (A) rebamipide and (B) furconazole tablets with G-DATs and $\mathrm{CCV}$

G-DAT and CCVs from pharmacokinetic parameters of 7 rebamipide and 5 furconazole tablets was calculated.

peutic effect using generic drugs, which had an uneven CCV graph even if G-DAT showed nearly equal to 1 , is not equivalent to that of the original drug because of variances in the kinetic parameters between original and generic drugs. We shall consider to monitor the therapeutic effect using the generic drug selected by the proposed method.

\section{Discussion}

The need to reduce anxieties about taking generic drugs was emphasized in the report entitled "Survey research for safety use of generic drugs" announced by the Ministry of Health, Labour, and Welfare in Japan on November 25, 2009. Patients need to select generic drugs manufactured based on an original medicine. It is desirable for pharmacists to see at least an objective criterion for assisting patients to select a generic drug. Therefore, some criteria are required to compare generic drugs. The use of the pharmacokinetic data of generic drugs in the bioequivalence study for di- rect comparison raises a statistical problem on a different target for comparison. To solve this problem, we proposed a statistical comparison using G-DAT, calculated from the rate of the coefficient of variance (CCV) between generic and original drugs based on the vector space method. Our proposed method is applicable to drugs presented pharmacokinetic parameters resulting from the bioequivalence study but is not to generic drugs of injectable drugs and most of external medications which are unnecessary for the bioequivalence study. The vector space method was applied to evaluate standardization and similarity of drug names by Tsuchiya et al. ${ }^{6}$ ) They expressed stem of a drug name as a vector and compared similarity of two particular drugs with an angle formed by two vectors and calculated with the cosine theorem. This method was applied to analyze relationship between similarity of drug names and confusion errors. ${ }^{7)}$

In this report, the quality variance of most generic drugs was evaluated to be little and in a de- 
fined area in the frequency distribution for GDATs (Figs. 1 and 2). The correlation between solubility and CV were analyzed with antihypertensive dihydropyridines (Table 3) and the result suggests that drugs with lower the solubilities have larger $\mathrm{CV}$. One and two generic drugs of rebamipide and furconazole, respectively, were found to be different from original drug in the GDAT histogram and the pharmacokinetic parameters-CCV plot as shown in Fig. 5 although these drugs passed the bioequivalence study and are put on the market. The results of this study provide a possible solution to reduce anxieties about taking generic drugs and may increase the prescription of generic drugs.

CVs of AUC and Cmax in weakly-basic and practically insoluble drugs tended to be higher than in soluble drugs. Weakly-basic and practically insoluble drugs dissolved in gastric acidic $\mathrm{pH}$ are likely to be precipitated and crystallized from near the duodenum to the upper region of small intestine by neutralization with pancreatic juice. The reason for the higher CVs is probably that drug trialists have different degrees of precipitation and crystallization of the drugs. Class II drugs show low solubility and high permeability in the BCS classification. Therefore, it is important to improve solubility and the rate of dissolution of class II drugs in drug formulation. ${ }^{8}$ These improvements seem to achieve lower CVs and result in stabilization of CCVs. Therefore, our proposed evaluation method using G-DAT and CCVs shown in Fig. 4 can be a useful tool for judging whether generic class II drugs are adequately formulated to improve solubility and rate of dissolution.

Our method for evaluating the quality of generic drugs proposed in this report can be applied to drugs not only in Japan but also in other countries if pharmacokinetic parameters of the drugs are available.

\section{Acknowledgment}

We gratefully thank Nichi-Iko Pharmaceutical Co., Ltd. for kindly providing results of the dissolution test of several drugs.

\section{References}

1) Iijima $H$, Kamei M, Koshimizu T, Shiragami M, Evaluation of information for generic drugs based on importance and necessity, Yakugaku Zasshi, 2005, 125, 739-747.

2) Katoh H, Yoshii M, Ozawa K, Comparative study of drug efficacy and drug additives between generic drugs and original drugs, Yakugaku Zasshi, 2007, 127, 2035-2044.

3) Onda M, Kanematsu M, Kitamura T, Sakai T, Sakagami K, Tanaka K, Hamahata Y, Hirooka Y, Fujii K, Matsuda M, Miki H, Mashimo H, Hada R, Arakawa Y, Availability of drug information on bioequivalence of generic products--findings of graduate interns at a university pharmacy, Yakugaku Zasshi, 2007, 127, 1159-1166.

4) Harada K, Kawagoe K, Vector-space model modification and its physical structure for improving WWW serch efficiency, IPSJ Transaction, 1999, 40 (No. SIG6), 22-30.

5) Yu LX, Amidon GL, Polli JE, Zhao H, Mehta MU, Conner DP, Shah VP, Lesko LJ, Chen ML, Lee VH, Hussain AS, Biopharmaceutics classification system: the scientific basis for biowaiver extensions, Pharm Res, 2002, 19, 921-925.

6) Tsuchiya F, Kawamura N, Oh C, Hara A, Standardization and similarity deliberation of Drug-names, Japan Journal of Medical Informatics, 2001, 21, 5967.

7) Yamade Y, Haga S, Tsuchiya F, Shin H, Similarity of Drug Names and Confusion Errors: Laboratory Experiments with Students and Pharmacists, Cognitive Studies, 2006, 13, 80-95.

8) Takagi T, Yamashita S, Evaluation of solubility, "Physicochemical Characterization and Formulation Technologies for Poorly Soluble Drugs”, ed. by Kawakami K, CMC Publishing Tokyo, 2010, pp.1-20. 